\title{
Index to Volume 63: Book Reviewers
}

Daniel F. Austin, 93, 102, 216, 218, Ardeshir (Adi) B. Damania, 219 222, 320, 321, 323

Anbreen Bashir, 327

Dorothea Bedigian, 220, 446, 454

Brett M. Bennett, 221

Joost Bogemans, 97, 103, 221, 327

Mary Theresa

Bonhage-Freund, 101, 319, 451

Thomas Brendler, 447

Rainer W. Bussmann, 215

Alejandro Casas, 443

David A. Cleveland, 442

Walter R. Courtenay, Jr., 452
Jessica M. Dolan, 217

Eckart Eich, 444, 445

Mary Eubanks, 324, 441

Anil K. Goel, 96, 102, 327

Tasha Goldberg, 95

Douglas Goldman, 94

Alan Hamilton, 326

Neil A. Harriman, 225, 329, 450

Amy Keller, 449

Robert J. Krueger, 446

Aaron M. Lampman, 322

Pesach Lubinsky, 215, 322
Will C. McClatchey, 95

Kimberly E. Medley, 103, 325

Lytton John Musselman, 451

Michael Newberry, 453

My Lien Nguyen, 98, 99, 223

Concepción Obón, 223, 224

Diego Rivera, 223, 224

Claudia Segovia-Salcedo, 100

Stephen F. Siebert, 94, 329

Patrick Van Damme, 328, 448,449

Alejandra E. Vilela, 320

Justin K. Williams, 99 